\title{
Identification of global transcriptome abnormalities and potential biomarkers in eutopic endometria of women with endometriosis: A preliminary study
}

\author{
LUYANG ZHAO ${ }^{1,2}$, CHENGLEI GU ${ }^{1,3}$, MINGXIA YE ${ }^{1}$, ZHE ZHANG $^{1}$, \\ WEIDONG HAN ${ }^{4}$, WENSHENG FAN ${ }^{1}$ and YUANGUANG MENG ${ }^{1}$
}

\author{
${ }^{1}$ Department of Gynecology and Obstetrics, People's Liberation Army Medical School, \\ Chinese People's Liberation Army General Hospital, Beijing 100853; ${ }^{2}$ Department of Gynecology and Obstetrics, \\ Peking University People's Hospital, Beijing 100044; ${ }^{3}$ Department of Gynecology and Obstetrics, \\ the 309th Hospital of Chinese People's Liberation Army, Beijing 100853; ${ }^{4}$ Department of \\ Molecular Biology, Institute of Basic Medicine, People's Liberation Army Medical School, \\ Chinese People's Liberation Army General Hospital, Beijing 100853, P.R. China
}

Received March 23, 2017; Accepted April 13, 2017

DOI: $10.3892 /$ br.2017.902

\begin{abstract}
The etiology and pathophysiology of endometriosis remain unclear. The aim of the current study was to identify a candidate pathogenic gene, as well as potential biomarkers of endometriosis using messenger RNA (mRNA) sequencing (mRNA-seq). Twenty-three eutopic endometria from women with endometriosis and 20 endometria from control subjects were investigated. Eight eutopic endometria and five normal endometria were selected for mRNA-seq. Differentially expressed genes (DEGs) were identified and functional analysis was conducted. Validation of certain DEGs was performed in the remaining cases and control subjects by reverse transcription-quantitative polymerase chain reaction (RT-qPCR). A total of 72 DEGs (66 upregulated and 6 downregulated) were identified in samples from women with endometriosis and compared with the control subjects. High DEGs included those involved in various functions, such as extracellular matrix (ECM) remodeling, angiogenesis, cell proliferation and differentiation. Enriched by these DEGs, 100 Gene Ontology terms were identified as significantly important, particularly 'ECM' and 'endogenous stimulus'. Validation using RT-qPCR indicated that matrix metallopeptidase 11, dual specificity phosphatase 1, Fos proto-oncogeneand serpin family E member 1 were significantly upregulated and adenosine
\end{abstract}

Correspondence to: Dr Wensheng Fan or Dr Yuanguang Meng, Department of Gynecology and Obstetrics, People's Liberation Army Medical School, Chinese People's Liberation Army General Hospital, 28 Fuxing Road, Haidian, Beijing 100853, P.R. China E-mail: 455689429@qq.com

E-mail: meng6512@vip.sina.com

Key words: endometriosis, eutopic endometrium, messenger RNA, high-throughput nucleotide sequencing, biomarker deaminase 2 was significantly downregulated in the eutopic endometrium of patients with endometriosis. The identified DEGs may be involved in the pathogenesis of endometriosis and may be potential biomarkers in the eutopic endometrium. The current study provides a comprehensive, but preliminary insight for elucidating the mechanisms of endometriosis, which require further in-depth studies for confirmation.

\section{Introduction}

Endometriosis is characterized by the presence of endometrial tissue (glandular and stromal) abnormally outside the uterine cavity (1). According to certain statistics, there is an average of 10.4 years elapse from the first onset of symptoms to diagnosis, and $74 \%$ of patients receive at least one false diagnosis, which results in economic costs comparable with certain serious chronic diseases (2). Despite its significant impact on the quality of life and financial burden on patients, the etiology and pathophysiology of endometriosis remain unclear. Various theories have been proposed to explain the pathogenesis; however, none have interpreted it comprehensively (3). Sampson's retrograde menstruation theory is the most widely accepted, which proposes that fragments of the eutopic endometrium are implanted into the peritoneum or pelvic organs during menstruation through reflux via the fallopian tubes (4). Subsequently, extensive studies have been conducted to identify the differences between the eutopic and normal endometrium; indeed, various studies have demonstrated a distinct expression pattern in the eutopic endometrium compared with the normal endometrium, but the evidence is insufficient (5). In addition, laparoscopy remains the gold standard for the diagnosis of endometriosis and, to date, a histological examination is necessary for confirmation. However, this diagnostic method is a surgically invasive inspection requiring general anesthesia, which carries surgery-associated risks, such as hemorrhage, infection and adhesion formation, and requires an experienced surgeon (6). As a result of these drawbacks, 
non-invasive biomarkers using serum, urine and endometrial tissue as research samples have emerged; however, these current diagnostic tests for endometriosis lack sensitivity and specificity, and are difficult to repeat (7). Therefore, a thorough and comprehensive description of molecular differences between the eutopic endometrium in a patient with endometriosis and a control subject with a normal endometrium is essential to understand the pathogenesis of this disorder, and identify sensitivity and specificity biomarkers.

In the current study, it was hypothesized that the eutopic endometrium in endometriosis patients contains aberrant expression genes and exhibits dysregulated pathways that predispose itself to implant, invade and migrate outside the uterus. Messenger RNA (mRNA) sequencing was performed to detect the transcriptome expression profiling of eutopic endometrium in women with endometriosis compared with normal endometrium from healthy control subjects. Through global mRNA expression profiling, the aim was to identify candidate pathogenic genes and pathways that are implicated in the pathogenesis of endometriosis, as well as potential biomarkers of this common, clinically significant, but complex disorder.

\section{Materials and methods}

Ethics statement. The study protocol was approved by the Local Ethics Committee of Chinese People's Liberation Army (PLA) General Hospital (Beijing, China), and each patient was involved in the study after providing written informed consent.

Patient samples. Twenty-three eutopic endometria from patients with endometriosis were obtained from the Chinese PLA General Hospital between February and September 2016. Among them, eight eutopic endometria were randomly selected and prepared for mRNA sequencing (mRNA-seq), and the remaining 15 samples were used for validation. All patients were confirmed to have endometriosis by histological examination and diagnosed as being of moderate to severe stage (stage III-IV) according to the revised American Fertility Society (rAFS) classification (8) during laparoscopic surgery. None of the patients received hormone therapy prior to sampling. Twenty women without endometriosis, who underwent laparoscopic surgery for examination or hydrotubation, were included in the control group. Five endometria were randomly selected for sequencing analysis and 15 endometria were used for validation. Regions potentially exhibiting endometriotic lesions were confirmed as being negative by biopsy.

The eutopic and normal endometria were obtained via curettage prior to the laparoscopic procedure. Only patients in the secretory phase of the menstrual cycle, which was confirmed by the method of Noyes et al (9) were included in the study. There were no significant differences between the ages and body mass index values of the patient and control groups.

Tissue processing, RNA extraction and quality control. All tissue samples were divided into two parts: One-half was fixed and prepared for pathological examination to identify the endometrial phase of the menstrual cycle and the other half was placed in RNAlater solution (Sigma-Aldrich; Merck KGaA,
Darmstadt, Germany) at $4^{\circ} \mathrm{C}$ for $24 \mathrm{~h}$, and subsequently transferred to $-80^{\circ} \mathrm{C}$ until use. Total RNA was extracted using the single-step acid guanidinium thiocyanate-phenol-chloroform method (10). The quality and purity of RNA were examined using a Nanodrop 8000 spectrophotometer (Thermo Fisher Scientific, Inc., Waltham, MA, USA) and Bioanalyzer 2100 (Agilent Technologies, Inc., Santa Clara, CA, USA). Samples with RNA integrity number $\geq 8$ were included.

$m R N A$ sequencing and data analysis. Three micrograms of RNA per sample was prepared for library construction. The Ribo-Zero Gold kit (Epicentre; Illumina, Inc., San Diego, CA, USA) and NEB Next Ultra RNA Library Prep kit (New England BioLabs, Inc., Ipswich, MA, USA) were used for rRNA removal and library construction according to the manufacturer's instructions. For high-throughput sequencing, paired-end 150-bp sequencing of the cDNAs was performed using the Illumina HiSeq4000 system (Illumina, Inc.), which was conducted by Annoroad Genomics (Beijing, China). Raw data were processed with Perl scripts to ensure the quality of data used for further analysis. Bowtie2 (v2.2.3; https:// sourceforge.net/projects/bowtie-bio/files/bowtie2/) was used for building the genome index, and clean data was mapped to the human genome build (hg19) using Tophat (v2.0.12; https://tophat.cbcb.umd.edu/). Read counts of each gene were counted by HTSeq (v0.6.0; http://www-huber.embl. de/users/anders/HTSeq/doc/overview.html), and reads per $\mathrm{kb}$ of a gene per million reads (RPKM) were subsequently calculated to estimate the expression level of genes in each sample. DEGseq (v1.18.0; http://www.bioconductor. org/packages/release/bioc/html/DEGseq.html) was used for analyzing differentially expressed genes (DEGs) with the following parameters: False discovery rate (FDR) $\leq 0.05$ and fold-change $(\mathrm{FC}) \geq 2$ or $\leq 0.5$.

Reverse transcription-quantitative polymerase chain reaction $(R T-q P C R)$. Five mRNAs, including matrix metallopeptidase 11 (MMP-11; ENSG00000099953), dual specificity phosphatase 1 (DUSP1; ENSG00000120129), Fos proto-oncogene, AP-1 transcription factor subunit (FOS; ENSG00000170345), serpin family E member 1 (SERPINE1; ENSG00000106366), and adenosine deaminase 2 (ADA2; ENSG00000093072) were selected for validation analysis, and GAPDH served as an mRNA endogenous control. The primers are presented in Table I. cDNA synthesis was conducted using a RevertAid $^{\mathrm{TM}}$ First Strand cDNA Synthesis kit (Thermo Fisher Scientific, Inc.). The relative mRNA expression was determined by RT-qPCR according to the THUNDERBIRD ${ }^{\mathrm{TM}}$ SYBR qPCR Mix (Toyobo, Co., Ltd., Osaka, Japan). qRT-PCR was performed on an ABI PRISM 7500 (Applied Biosystems; Thermo Fisher Scientific, Inc.). The relative gene expression was calculated using ABI PRISM 7500 version 2.0.6 software (Applied Biosystems; Thermo Fisher Scientific, Inc.) according to the $2^{-\Delta \Delta C} \mathrm{q}$ method (11).

Functional analysis. To exploit the functional roles of DEGs, DAVID (https://david.ncifcrf.gov/home.jsp) was used, which integrated the Gene Ontology (GO) and Kyoto Encyclopedia of Genes and Genomes (KEGG) databases to analyze biological function. Finally, the enrichment values of the GO terms, 
Table I. Reverse transcription-quantitative polymerase chain reaction primers.

\begin{tabular}{lcll}
\hline Primer & Length, bp & \multicolumn{1}{c}{ Sequence } \\
\hline Matrix metallopeptidase 11 & 93 & $\begin{array}{l}\text { Forward: GCTGCCTTCCAGGATGCTGAT } \\
\text { Reverse: GCCTTCCAGAGCCTTCACCTT }\end{array}$ \\
Dual specificity phosphatase 1 & 85 & $\begin{array}{l}\text { Forward: GCCACCATCTGCCTTGCTTAC } \\
\text { Reverse TGCTTCGCCTCTGCTTCACA }\end{array}$ \\
Fos proto-oncogene, AP-1 transcription factor subunit & 245 & $\begin{array}{l}\text { Forward: CGAGATTGCCAACCTGCTGAAG } \\
\text { Reverse: CCATGCTGCTGATGCTCTTGAC }\end{array}$ \\
Serpin family E member 1 & 262 & $\begin{array}{l}\text { Forward: TTCAGGCTGACTTCACGAGT } \\
\text { Reverse: CCAGATGAAGGCGTCTTCC }\end{array}$ \\
Adenosine deaminase 2 & 237 & $\begin{array}{l}\text { Forward: GGCTGTCATCGCAGAATCCATC } \\
\text { Reverse: AGCATCAGAGCATCCAGAATGTTC }\end{array}$ \\
\hline
\end{tabular}

Table II. Summary of the mRNA sequencing data following filtering and mapping.

\begin{tabular}{lccrrrc}
\hline Sample & Total raw reads & Total Q30 $(\%)$ & Total clean reads & Mapped reads & Unique map reads & MultiMap reads \\
\hline EU1 & $108,063,442$ & 94.38 & $106,300,690$ & $99,490,109$ & $98,103,400$ & $1,386,709$ \\
EU5 & $115,796,574$ & 94.93 & $114,245,924$ & $108,073,952$ & $106,633,173$ & $1,440,779$ \\
EU6 & $88,538,384$ & 94.76 & $87,285,608$ & $81,742,080$ & $80,563,047$ & $1,179,033$ \\
EU7 & $125,048,606$ & 94.97 & $123,155,952$ & $116,419,348$ & $114,821,450$ & $1,597,898$ \\
EU11 & $112,650,384$ & 94.35 & $110,637,684$ & $102,884,399$ & $101,501,805$ & $1,382,594$ \\
EU18 & $89,731,074$ & 94.42 & $88,482,524$ & $83,389,862$ & $82,191,074$ & $1,198,788$ \\
EU19 & $105,616,842$ & 94.57 & $104,296,544$ & $97,924,454$ & $96,502,461$ & $1,421,993$ \\
EU21 & $112,190,626$ & 94.68 & $110,717,296$ & $103,453,282$ & $102,134,657$ & $1,318,625$ \\
N2 & $119,170,846$ & 94.89 & $117,104,548$ & $109,135,053$ & $107,668,064$ & $1,466,989$ \\
N8 & $100,224,148$ & 95.00 & $98,937,330$ & $92,856,399$ & $91,660,813$ & $1,195,586$ \\
N12 & $95,701,846$ & 94.50 & $94,451,810$ & $88,290,492$ & $87,035,145$ & $1,255,347$ \\
N16 & $106,411,788$ & 94.92 & $104,457,624$ & $97,033,960$ & $95,631,762$ & $1,402,198$ \\
N19 & $116,983,022$ & 93.76 & $114,885,072$ & $107,817,828$ & $106,214,767$ & $1,603,061$ \\
\hline
\end{tabular}

obtained using the hypergeometric test, were considered significantly enriched when the q-value (adjusted as a P-value) was 0.05 .

\section{Results}

mRNA filtering and mapping. mRNA sequencing generated $1,396,127,582$ reads, with an average of 107,204,492 reads per sample in the eutopic endometrium group and 107,698,330 reads per sample in the normal endometrium group. A fastQC quality test demonstrated that 1,374,958,606 (98.48\%) reads had a Q-score $\geq 30$, and thus were considered for further analyses. Of these reads, $93.71 \%$ were mapped to the hg19 and $98.61 \%$ were uniquely aligned. The detailed filtering and mapping data are presented in Table II.

Identification of DEGs and RT-qPCR findings. Among the 13 samples, there were 40,576 mRNAs with an RPKM value of 1 in at least one sample. The transcriptome expression profiling of eutopic endometria and normal endometria exhibited very similar expression levels in the evaluated mRNAs, which directly indicated the homology of the two sequenced groups
(Fig. 1A). On the basis of the above-mentioned criteria of DEGs, 72 DEGs were identified with 66 upregulated genes and 6 downregulated genes (Table III and Fig. 1B).

Two DEGs, ADA2 and MMP-11, were significantly different in the current study, but were not previously selected for further validation. FOS, which exhibited contradictory results in previous studies, was selected. In addition, two DEGs, SERPINE1 and DUSP1, which have been associated with endometriosis in cell lines and animal models, but have not been reported in human tissue, were simultaneously selected. Although the counts of DEGs in mRNA sequencing were relatively low in the two groups, the RT-qPCR analysis indicated easily detectable expression levels. Data analysis indicated that the results from RT-qPCR were consistent with the mRNA sequencing data (Fig. 2).

Functional analysis. To gain an overall understanding of the functional roles in these DEGs, GO term and KEGG pathway analysis were conducted. The results revealed that significantly enriched GO terms under the cellular component $(\mathrm{CC})$ category were extracellular matrix (ECM; 
Table III. Differentially expressed genes $(n=72)$ in eutopic endometrium samples from women with endometriosis versus healthy control subjects.

\begin{tabular}{|c|c|c|c|c|}
\hline Gene symbol & Ensemble ID & Change & Fold-change & False discovery rate \\
\hline RP11-319E12.2 & ENSG00000251459 & Upregulated & 120.99 & 2.32E-07 \\
\hline IGFBP1 & ENSG00000146678 & Upregulated & 115.01 & $1.79 \mathrm{E}-03$ \\
\hline SERPINB2 & ENSG00000197632 & Upregulated & 72.95 & $1.51 \mathrm{E}-04$ \\
\hline FOSB & ENSG00000125740 & Upregulated & 66.12 & $2.01 \mathrm{E}-19$ \\
\hline EREG & ENSG00000124882 & Upregulated & 52.02 & $2.05 \mathrm{E}-09$ \\
\hline MMP27 & ENSG00000137675 & Upregulated & 40.93 & 1.69E-04 \\
\hline MMP10 & ENSG00000166670 & Upregulated & 31.41 & $4.01 \mathrm{E}-04$ \\
\hline LEFTY2 & ENSG00000143768 & Upregulated & 30.59 & 1.37E-08 \\
\hline WIF1 & ENSG00000156076 & Upregulated & 30.56 & 1.38E-04 \\
\hline CDC20B & ENSG00000164287 & Upregulated & 25.21 & $6.06 \mathrm{E}-09$ \\
\hline LRRC15 & ENSG00000172061 & Upregulated & 25.20 & $3.17 \mathrm{E}-24$ \\
\hline LINC01411 & ENSG00000249306 & Upregulated & 24.95 & 7.67E-03 \\
\hline RPL10P9 & ENSG00000233913 & Upregulated & 21.83 & $3.10 \mathrm{E}-02$ \\
\hline IGKV1-12 & ENSG00000243290 & Upregulated & 21.19 & $2.04 \mathrm{E}-05$ \\
\hline FAM159B & ENSG00000145642 & Upregulated & 20.34 & 2.34E-02 \\
\hline INHBA & ENSG00000122641 & Upregulated & 15.33 & $1.19 \mathrm{E}-15$ \\
\hline FOXN4 & ENSG00000139445 & Upregulated & 14.90 & $1.71 \mathrm{E}-02$ \\
\hline RGS1 & ENSG00000090104 & Upregulated & 14.80 & $1.19 \mathrm{E}-15$ \\
\hline MMP3 & ENSG00000149968 & Upregulated & 14.71 & 4.40E-02 \\
\hline FOS & ENSG00000170345 & Upregulated & 14.54 & $2.55 \mathrm{E}-24$ \\
\hline IGHV1-2 & ENSG00000211934 & Upregulated & 14.08 & 3.82E-04 \\
\hline NR4A1 & ENSG00000123358 & Upregulated & 13.80 & $8.80 \mathrm{E}-17$ \\
\hline EGR3 & ENSG00000179388 & Upregulated & 13.24 & $1.19 \mathrm{E}-15$ \\
\hline VGFA & ENSG00000112715 & Upregulated & 13.10 & $8.07 \mathrm{E}-17$ \\
\hline RP11-459E5.1 & ENSG00000253125 & Upregulated & 12.77 & 8.50E-06 \\
\hline ARC & ENSG00000198576 & Upregulated & 12.72 & $3.82 \mathrm{E}-04$ \\
\hline EPYC & ENSG00000083782 & Upregulated & 12.28 & $4.50 \mathrm{E}-02$ \\
\hline KCNF1 & ENSG00000162975 & Upregulated & 10.88 & $1.33 \mathrm{E}-02$ \\
\hline AREG & ENSG00000109321 & Upregulated & 9.49 & $6.67 \mathrm{E}-03$ \\
\hline FOSL1 & ENSG00000175592 & Upregulated & 9.42 & $1.87 \mathrm{E}-08$ \\
\hline VGF & ENSG00000128564 & Upregulated & 9.14 & $3.29 \mathrm{E}-02$ \\
\hline NPTX1 & ENSG00000171246 & Upregulated & 9.11 & $2.50 \mathrm{E}-02$ \\
\hline ATF3 & ENSG00000162772 & Upregulated & 8.99 & 3.83E-08 \\
\hline SERPINE1 & ENSG00000106366 & Upregulated & 8.17 & $2.00 \mathrm{E}-04$ \\
\hline IL11 & ENSG00000095752 & Upregulated & 7.97 & 7.57E-04 \\
\hline IGFN1 & ENSG00000163395 & Upregulated & 7.79 & 2.78E-05 \\
\hline ASIC2 & ENSG00000108684 & Upregulated & 7.40 & $1.31 \mathrm{E}-02$ \\
\hline NR4A3 & ENSG00000119508 & Upregulated & 7.30 & 4.57E-06 \\
\hline CRYGN & ENSG00000127377 & Upregulated & 7.25 & $9.11 \mathrm{E}-03$ \\
\hline AP000349.2 & ENSG00000280178 & Upregulated & 7.07 & $4.06 \mathrm{E}-13$ \\
\hline MTUS2 & ENSG00000132938 & Upregulated & 6.87 & $5.42 \mathrm{E}-03$ \\
\hline $\mathrm{ZCCHC12}$ & ENSG00000174460 & Upregulated & 6.82 & $1.05 \mathrm{E}-03$ \\
\hline MMP11 & ENSG00000099953 & Upregulated & 6.77 & $5.21 \mathrm{E}-13$ \\
\hline ARSI & ENSG00000183876 & Upregulated & 6.46 & $9.06 \mathrm{E}-03$ \\
\hline LOC101929415 & ENSG00000254254 & Upregulated & 5.90 & $4.56 \mathrm{E}-02$ \\
\hline RP11-613D13.8 & ENSG00000244953 & Upregulated & 5.72 & 2.98E-03 \\
\hline KRT17 & ENSG00000128422 & Upregulated & 5.56 & 9.34E-05 \\
\hline EGR1 & ENSG00000120738 & Upregulated & 5.47 & $1.43 \mathrm{E}-09$ \\
\hline CYR61 & ENSG00000142871 & Upregulated & 5.38 & 2.70E-09 \\
\hline GEM & ENSG00000164949 & Upregulated & 4.72 & 7.61E-06 \\
\hline C11orf96 & ENSG00000187479 & Upregulated & 4.57 & $2.05 \mathrm{E}-04$ \\
\hline
\end{tabular}


Table III. Continued.

\begin{tabular}{llccc}
\hline Gene symbol & \multicolumn{1}{c}{ Ensemble ID } & Change & Fold-change & False discovery rate \\
\hline PTHLH & ENSG00000087494 & Upregulated & 4.51 & $3.73 \mathrm{E}-02$ \\
EGR2 & ENSG00000122877 & Upregulated & 4.41 & $1.33 \mathrm{E}-02$ \\
DUSP1 & ENSG00000120129 & Upregulated & 4.35 & $2.05 \mathrm{E}-04$ \\
LOC102724428 & ENSG00000275993 & Upregulated & 4.35 & $8.21 \mathrm{E}-03$ \\
PAMR1 & ENSG00000149090 & Upregulated & 4.23 & $1.34 \mathrm{E}-03$ \\
TNFRSF12A & ENSG00000006327 & Upregulated & 3.83 & $5.70 \mathrm{E}-03$ \\
DUSP5 & ENSG00000138166 & Upregulated & 3.66 & $1.60 \mathrm{E}-03$ \\
SLC47A1 & ENSG00000142494 & Upregulated & 3.62 & $8.33 \mathrm{E}-03$ \\
ADAMTS16 & ENSG00000145536 & Upregulated & 3.39 & $1.45 \mathrm{E}-02$ \\
CAB39L & ENSG00000102547 & Upregulated & 3.38 & $6.55 \mathrm{E}-03$ \\
HES1 & ENSG00000114315 & Upregulated & 3.23 & $1.60 \mathrm{E}-03$ \\
HTRA3 & ENSG00000170801 & Upregulated & 3.17 & $6.92 \mathrm{E}-03$ \\
LTBP2 & ENSG00000119681 & Upregulated & 2.92 & $4.88 \mathrm{E}-02$ \\
LOC284454 & ENSG00000267519 & Upregulated & 2.89 & $4.03 \mathrm{E}-02$ \\
RGCC & ENSG00000102760 & Upregulated & 2.74 & $2.98 \mathrm{E}-03$ \\
LRRC26 & ENSG00000184709 & Downregulated & 109.57 & $5.10 \mathrm{E}-08$ \\
S100A7 & ENSG00000143556 & Downregulated & 40.68 & $8.21 \mathrm{E}-03$ \\
PWP2 & ENSG00000241945 & Downregulated & 18.29 & $6.54 \mathrm{E}-03$ \\
GUCY1B2 & ENSG00000123201 & Downregulated & 11.64 & $6.54 \mathrm{E}-03$ \\
CTD-2384B11.2 & ENSG00000225407 & Downregulated & 7.54 & $1.30 \mathrm{E}-02$ \\
ADA2 & ENSG00000093072 & Downregulated & 3.23 & \\
\hline
\end{tabular}

A

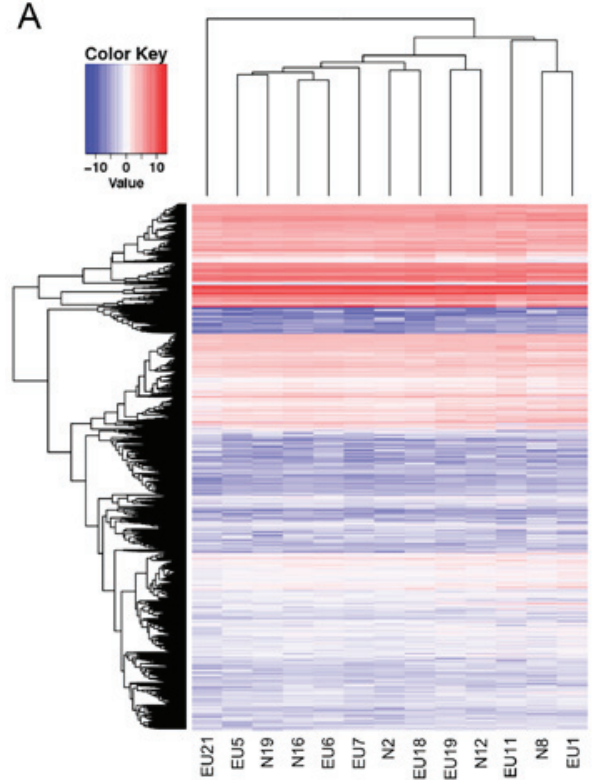

B

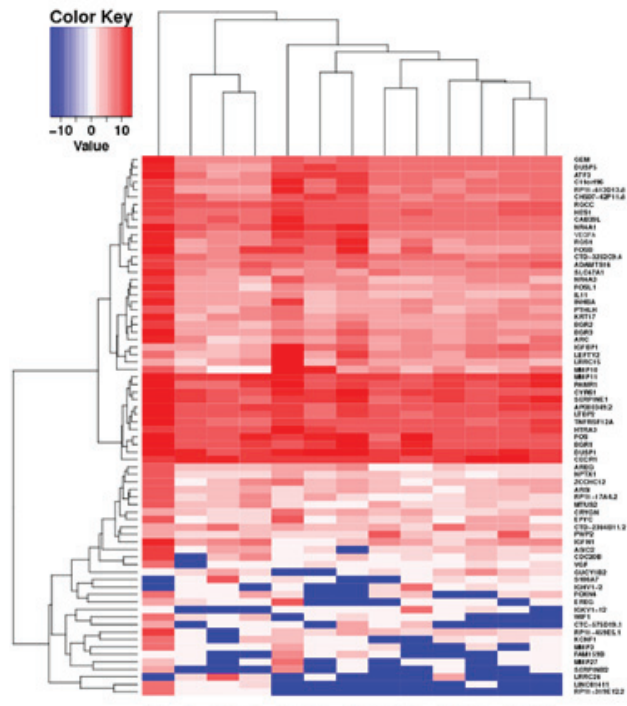

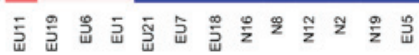

Figure 1. Hierarchical clustering analysis. (A) Unsupervised hierarchical clustering of the 40,576 expressed mRNAs and (B) supervised hierarchical clustering analysis of 72 differentially expressed genes between the eutopic endometrium samples from endometriosis patients and healthy control subjects.

GO:0031012), proteinaceous ECM (GO:0005578) and extracellular space (GO:0005615). The molecular function (MF) category included nine enriched terms, particularly in metalloendopeptidase activity (GO:0004222), growth factor activity (GO:0008083), and RNA polymerase II core promoter proximal region sequence-specific DNA binding transcription factor activity (GO:0000982). The biological process (BP) category contained 88 enriched terms, the top three of which were response to endogenous stimulus (GO:0009719), cellular response to endogenous stimulus (GO:0071495) and response to cyclic adenosine monophosphate (GO:0051591). In addition, certain GO terms that are commonly observed in tumor-like diseases were also significantly enriched in the current results, such as growth (GO:0040007), angiogenesis (GO:0001525) 


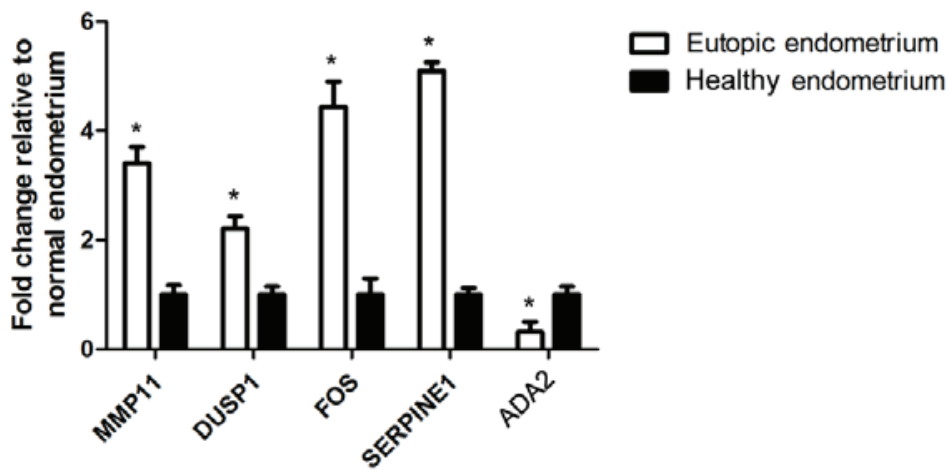

Figure 2. The expression levels of five selected mRNAs in eutopic endometrium samples from women with endometriosis ( $\mathrm{n}=15)$ and healthy control endometrial tissue samples $(\mathrm{n}=15)$ following reverse transcription-quantitative polymerase chain reaction. ${ }^{*} \mathrm{P}<0.01$ vs. healthy endometrium.

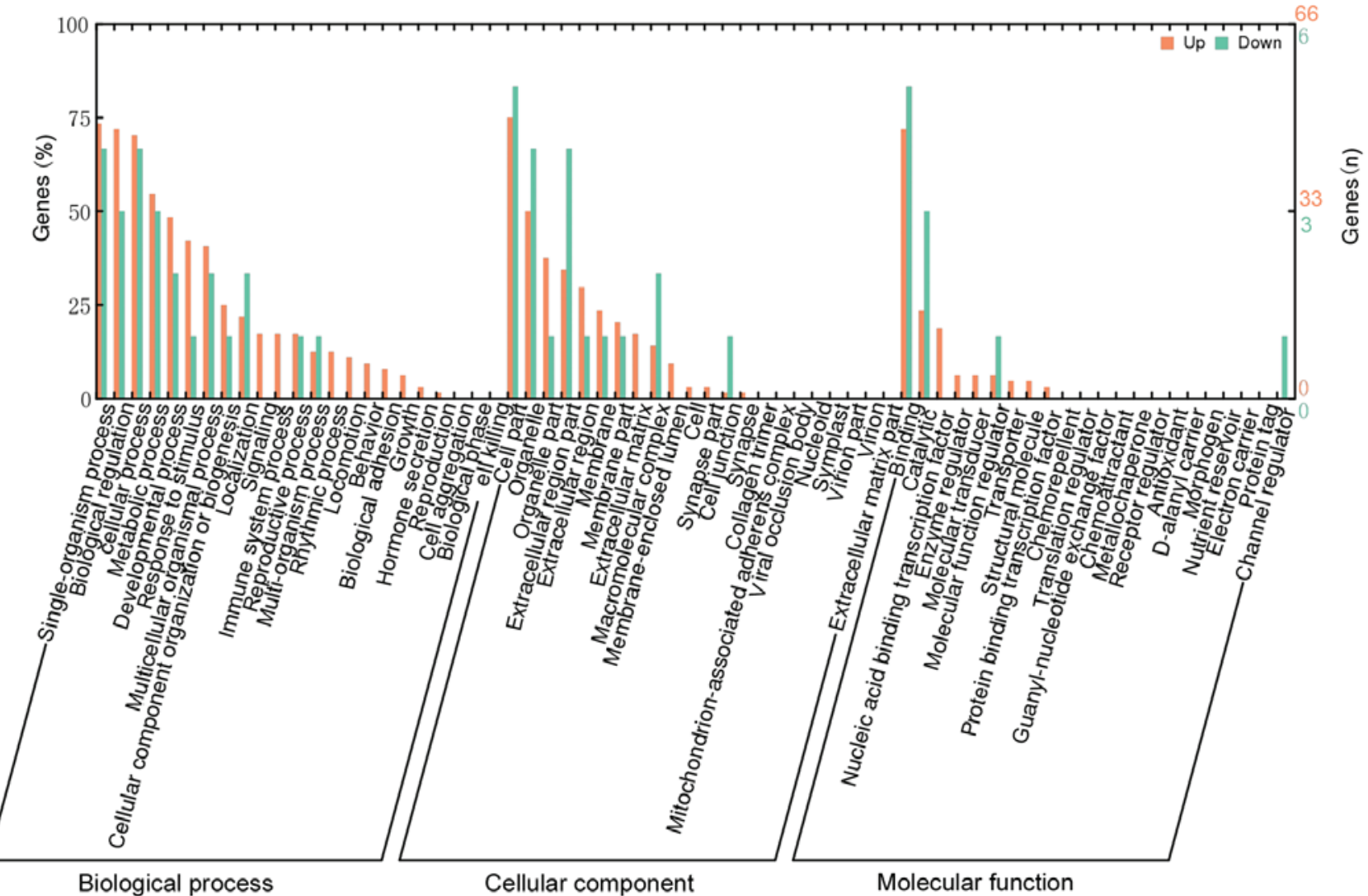

Figure 3. Gene Ontology annotations for the differentially expressed genes in the eutopic endometrium samples of women with endometriosis compared with the healthy control subjects. Upregulated genes are presented in red while downregulated genes are in green.

and cell migration (GO:0016477). These results are presented in Fig. 3 and Table IV. Due to the limited number of DEGs, none of the pathways were identified to be significantly enriched in the KEGG analysis with the above-mentioned thresholds.

\section{Discussion}

To date, the majority of studies that focused on the eutopic endometrium of women with endometriosis were hypothesis-based studies, which evaluated a limited number of previously susceptible genes (7). A recent systematic review from 1984 to 2010 summarized $>200$ potential endometrial biomarkers in the endometrium, but did not identify a standard biomarker in a clinical study (12). In addition, various studies using a microarray-based method have identified hundreds of potential pathogenic genes and pathways; however, these studies present few overlapping results (13-15). This inconsistency may be caused by various factors, including a small number of samples, poorly defined controls, different rAFS stages, different phases of the menstrual cycle, methodology limitations, various types of endometriosis, and interference by coexistent diseases. Therefore, future studies should continue to search for the important DEGs and focus on these confounding factors. To the best of our knowledge, the current study is the first to present the genome-wide gene expression profiling of the eutopic endometrium in women with endometriosis using 
Table IV. Top 10 enriched GO terms of DEGs in the endometrium from women with endometriosis compared with healthy control subjects.

\begin{tabular}{|c|c|c|c|c|c|}
\hline Category & GO ID & Description & $\mathrm{Q}$ value & $\mathrm{n}$ & Involved DEGs \\
\hline BP & GO:0009719 & Response to endogenous stimulus & 4.47E-07 & 21 & $\begin{array}{l}\text { CAB39L, SERPINE1, AREG, HES1, NR4A3, } \\
\text { LTBP2, DUSP1, EGR1, INHBA, EGR2, } \\
\text { NR4A1, EREG, FOSB, VGF, LEFTY2, } \\
\text { IGFBP1, MMP3, HTRA3, FOSL1, } \\
\text { EGR3, VEGFA }\end{array}$ \\
\hline BP & GO:0071495 & $\begin{array}{l}\text { Cellular response to endogenous } \\
\text { stimulus }\end{array}$ & $1.54 \mathrm{E}-06$ & 17 & $\begin{array}{l}\text { CAB39L, SERPINE1, HES1, LTBP2, DUSP1, } \\
\text { EGR1, INHBA, EGR2, NR4A1, EREG, } \\
\text { FOSB, LEFTY2, IGFBP1, MMP3, HTRA3, } \\
\text { EGR3, VEGFA }\end{array}$ \\
\hline BP & GO:0051591 & $\begin{array}{l}\text { Response to cyclic adenosine } \\
\text { monophosphate }\end{array}$ & $1.54 \mathrm{E}-06$ & 8 & $\begin{array}{l}\text { AREG, DUSP1, EGR1, EGR2, FOSB, VGF, } \\
\text { FOSL1, EGR3 }\end{array}$ \\
\hline $\mathrm{CC}$ & GO:0031012 & Extracellular matrix & $4.5 \mathrm{E}-06$ & 11 & $\begin{array}{l}\text { EPYC, MMP11, SERPINE1, LTBP2, MMP27, } \\
\text { CYR61, LEFTY2, ADAMTS16, MMP3, } \\
\text { MMP10, AP000349.2 }\end{array}$ \\
\hline BP & GO:0001525 & Angiogenesis & $1.2 \mathrm{E}-05$ & 9 & $\begin{array}{l}\text { TNFRSF12A, RGCC, SERPINE1, NR4A1, } \\
\text { EREG, CYR61, S100A7, EGR3, VEGFA }\end{array}$ \\
\hline $\mathrm{BP}$ & GO:0046683 & Response to organophosphorus & $1.49 \mathrm{E}-05$ & 8 & $\begin{array}{l}\text { AREG, DUSP1, EGR1, EGR2, FOSB, VGF, } \\
\text { FOSL1, EGR3 }\end{array}$ \\
\hline BP & GO:0014074 & $\begin{array}{l}\text { Response to purine-containing } \\
\text { compound }\end{array}$ & $1.95 \mathrm{E}-05$ & 8 & $\begin{array}{l}\text { AREG, DUSP1, EGR1, EGR2, FOSB, VGF, } \\
\text { FOSL1, EGR3 }\end{array}$ \\
\hline $\mathrm{BP}$ & GO:0048646 & $\begin{array}{l}\text { Anatomical structure formation } \\
\text { involved in morphogenesis }\end{array}$ & $3.16 \mathrm{E}-05$ & 17 & $\begin{array}{l}\text { TNFRSF12A, PTHLH, RGCC, SERPINE1, } \\
\text { NR4A3, DUSP1, INHBA, EGR2, NR4A1, } \\
\text { EREG, DUSP5, FOXN4, CYR61, S100A7, } \\
\text { ADAMTS16, EGR3, VEGFA }\end{array}$ \\
\hline BP & GO:0010243 & $\begin{array}{l}\text { Response to organonitrogen } \\
\text { compound }\end{array}$ & $5.42 \mathrm{E}-05$ & 14 & $\begin{array}{l}\text { CAB39L, AREG, HES1, NR4A3, DUSP1, } \\
\text { EGR1, EGR2, EREG, FOSB, VGF, } \\
\text { IGFBP1, MMP3, FOSL1, EGR3 }\end{array}$ \\
\hline BP & GO:0009725 & Response to hormone stimulus & $8.50 \mathrm{E}-05$ & 14 & $\begin{array}{l}\text { CAB39L, AREG, HES1, NR4A3, DUSP1, } \\
\text { EGR1, INHBA, EGR2, EREG, FOSB, } \\
\text { VGF, IGFBP1, FOSL1, EGR3 }\end{array}$ \\
\hline
\end{tabular}

GO, Gene Ontology; DEG, differentially expressed gene; BP, biological process; CC, cellular component.

a transcriptome sequencing technique. Considering that the genome profiling of normal endometria demonstrated marked molecular differences between samples obtained from the proliferative and secretory phases of the menstrual cycle, only mid- and late-secretory phase endometria were investigated in the patient and control groups, which represents most closely the reflux endometrium. To avoid other confounding factors, the endometriosis patients included were restricted to those in the moderate to severe stages (stages III-IV) and only those patients with ovarian endometriosis without combined diseases were enrolled. Finally, 72 DEGs enriched in 100 functional GO terms were identified. The top enriched terms in each category were ECM (GO:0031012) in CC, metalloendopeptidase activity (GO:0004222) in MF, and cellular response to endogenous stimulus (GO:0009719) in BP. Notably, various DEGs may be candidates for potential biomarkers in the eutopic endometrium of women with endometriosis.

It has been reported that human endometrium undergoes cyclic tissue remodeling during the menstrual period, during which several MMPs and ECM-associated proteins are activated (16). These proteins are suggested to facilitate the degradation and invasion of ECM and facilitate with the attachment of reflux endometrial tissue to the peritoneum and ovarian surface. In the current study, four MMP members, MMP-3, MMP-10, MMP-11, and MMP-27, were identified as upregulated in eutopic endometrium (17). Gilabert-Estellés et al (18) and Ramón et al (19) demonstrated that eutopic endometria from women with endometriosis have increased expression levels of MMP-3, which is consistent with the present result. Uzan et al (20) examined the immunohistochemical expression of MMP-11, although no difference was identified between the patient and control groups. Cominelli et al (21) suggested that MMP-27 is maximally expressed during the menstrual phase in the normal endometrium and no difference in ectopic versus eutopic endometria was observed; however, the authors did not compare between eutopic and normal endometria. Prior studies reported that the overexpression of SERPINE1 (also termed PAI-1) may result in the impairment of the fibrinolytic system, rendering the woman prone to endometriosis (22). Braza-Boills et al (23) demonstrated that the protein expression 
levels of SERPINE1 were significantly higher in endometriotic lesions than in control endometrial tissue samples, but identified no difference between eutopic and control endometria. Unlike other members mentioned above, MMP-10, epiphycan, latent transforming growth factor $\beta$ binding protein 2 , cysteine rich angiogenic inducer 61, left-right determination factor 2, ADAM metallopeptidase with thrombospondin type 1 motif 16 , and AP000349.2 have received little attention in endometriosis research and therefore require further confirmation.

The FOS gene family comprises four members, namely FOS, FosB proto-oncogene, AP-1 transcription factor subunit (FOSB), FOS like 1, AP-1 transcription factor subunit (FOSL1) and FOSL2. Their encoded proteins dimerize with the Jun family members to form the group of AP-1 proteins, and are involved in various physiological and pathological processes, such as cell proliferation, apoptosis, and differentiation and transformation (24). In the present study, abnormally high expression levels of FOS, FOSB, and FOSL1 were observed in eutopic endometria from patients with endometriosis, which were predominantly enriched in response to endogenous stimulus terms, nucleic acid-binding transcription factor activity terms, and response to hormone stimulus terms. FOS, as an early response gene, is critical in estrogen-mediated proliferation of endometrial cells (25). Pan et al (26) reported that FOS protein expression levels in eutopic and ectopic endometria samples from females with endometriosis were significantly higher than those in the endometria samples from healthy control subjects; however, the findings of Morsch et al (27) were not similar. Therefore, the FOS gene was selected to validate RT-qPCR in the present study, and the result was consistent with the results of Pan et al (26). To the best of our knowledge, the association between FOSB and FOSL1, and endometriosis have not yet been reported. As with FOS, the early growth response (EGR) family of transcription regulatory factors was predicted to be key in cellular growth and differentiation (28). Three members, EGR1, EGR2 and EGR3 were identified to be highly expressed by sequencing, which were also significantly enriched in response to endogenous stimulus terms, response to gonadotropin stimulus terms, and growth terms. Birt et al (29) reported the overexpression of EGR1 in endometriotic animal models and inferred that EGR1 may affect downstream protease pathways impeding ovulation in endometriosis. The roles of EGR1and EGR3 in angiogenesis has also recently been recognized and were considered to regulate certain important angiogenic factors, such as vascular endothelial growth factor A (VEGFA), fibroblast growth factor 2, and C-X-C motif chemokine ligand 1. Angiogenesis is considered to be pivotal to the implant and growth of endometriotic lesions in the pelvic microenvironment (30). The endometrium, which contains robust stem cell populations and striking regenerative ability, is a rich source of angiogenic factors (31). In the present study, enriched angiogenesis and blood vessel development terms were also observed. Aberrant upregulation of VEGFA in eutopic endometria demonstrated concordance with two earlier results: Taylor et al (32) emphasized the importance of VEGFA in the endometrium of women with endometriosis, as it may be activated by inflammatory-, oxidative-, hormonal- and endoplasmic reticulum-stress signals. Bourlev et al (33) reported a high expression level of VEGFA in the eutopic endometrium of women with endometriosis, as well as high concentrations of VEGFA in the peritoneal fluid. In addition, dysregulation of various angiogenic factors, including regulator of cell cycle, TNF receptor superfamily member $12 \mathrm{~A}$, nuclear receptor subfamily 4 group A member 1, epiregulin, cysteine rich angiogenic inducer 61, and S100 calcium binding protein A7, was observed in the present study in women with endometriosis when compared with those without endometriosis. Although these preliminary data require further characterization, the current findings provide novel information for future experimental studies.

In addition to gaining an improved understanding of pathogenesis, the present study attempted to identify various potential biomarkers. Although certain studies have questioned the unpleasant sensation of endometrial biopsy, the majority of participants are willing to undergo the procedure (34). In the current study, elevated expression levels of ADA2, MMP-11, FOS, SERPINE1, and DUSP1 in women with endometriosis were revealed by mRNA sequencing and RT-qPCR and, thus, these genes were considered as candidate biomarkers. Due to the limited sample size, the sensitivity, specificity, and receiver operating characteristic curve analysis were not calculated for a diagnostic test of endometriosis, which would be vital for a large sample-size study in future.

Despite the novel results, there were limitations of the current study. The primary limitation of the study is the relatively small sample size. In addition, it is difficult to evaluate whether the control endometria are from completely healthy women, as $\sim 6 \%$ of endometriotic lesions are only visible under a microscope, and these women macroscopically presented a normal appearance (35). In addition, these preliminary results require validation by downstream experiments.

In conclusion, to the best of our knowledge, the current study presents the first genome-wide gene expression profile of eutopic endometria from women with endometriosis using a high-throughput sequencing technique. Seventy-two DEGs in eutopic endometria from women with endometriosis were compared with normal endometria from control subjects. GO analysis further revealed the important roles of these DEGs in the pathogenesis of endometriosis. Five genes, including MMP-11, DUSP1, FOS, SERPINE1, and ADA2 were further confirmed by RT-qPCR, and the results were consistent with the mRNA sequencing, indicating that these genes may present as novel biomarkers in the endometrium of women with endometriosis. The current study provides a comprehensive, but preliminary insight into elucidating the underlying mechanisms of this complex disorder, which merits further in-depth studies for confirmation.

\section{Acknowledgements}

The current study was funded by the National Natural Science Foundation of China (grant no. 81571411).

\section{References}

1. Kennedy S, Bergqvist A, Chapron C, D'Hooghe T, Dunselman G Greb R, Hummelshoj L, Prentice A and Saridogan E; ESHRE Special Interest Group for Endometriosis and Endometrium Guideline Development Group: ESHRE guideline for the diagnosis and treatment of endometriosis. Hum Reprod 20: 2698-2704, 2005. 
2. Hudelist G, Fritzer N, Thomas A, Niehues C, Oppelt P, Haas D, Tammaa A and Salzer H: Diagnostic delay for endometriosis in Austria and Germany: Causes and possible consequences. Hum Reprod 27: 3412-3416, 2012

3. Sourial S, Tempest N and Hapangama DK: Theories on the pathogenesis of endometriosis. Int J Reprod Med 2014: 179515, 2014

4. Sampson JA: Metastatic or Embolic Endometriosis, due to the Menstrual Dissemination of Endometrial Tissue into the Venous Circulation. Am J Pathol 3: 93-110.43, 1927.

5. Liu $\mathrm{H}$ and Lang JH: Is abnormal eutopic endometrium the cause of endometriosis? The role of eutopic endometrium in pathogenesis of endometriosis. Med Sci Monit 17: RA92-RA99, 2011

6. Lattarulo S, Pezzolla A, Fabiano G and Palasciano N: Intestinal endometriosis: Role of laparoscopy in diagnosis and treatment. Int Surg 94: 310-314, 2009

7. Fassbender A, Vodolazkaia A, Saunders P, Lebovic D, Waelkens E, De Moor B and D'Hooghe T: Biomarkers of endometriosis. Fertil Steril 99: 1135-1145, 2013.

8. American Society for Reproductive: Revised American Society for Reproductive Medicine classification of endometriosis: 1996 Fertil Steril 67: 817-821, 1997.

9. Noyes RW, Hertig AT and Rock J: Dating the endometrial biopsy. Am J Obstet Gynecol 122: 262-263, 1975.

10. Chomczynski P and Sacchi N: The single-step method of RNA isolation by acid guanidinium thiocyanate-phenol-chloroform extraction: Twenty-something years on. Nat Protoc 1: 581-585, 2006.

11. Livak KJ and Schmittgen TD: Analysis of relative gene expression data using real-time quantitative PCR and the 2(-Delta Delta C(T)) Method. Methods 25: 402-408, 2001.

12. May KE, Villar J, Kirtley S, Kennedy SH and Becker CM: Endometrial alterations in endometriosis: A systematic review of putative biomarkers. Hum Reprod Update 17: 637-653, 2011.

13. Burney RO, Talbi S, Hamilton AE, Vo KC, Nyegaard M, Nezhat CR, Lessey BA and Giudice LC: Gene expression analysis of endometrium reveals progesterone resistance and candidate susceptibility genes in women with endometriosis. Endocrinology 148: 3814-3826, 2007.

14. Sherwin JR, Sharkey AM, Mihalyi A, Simsa P, Catalano RD and D'Hooghe TM: Global gene analysis of late secretory phase, eutopic endometrium does not provide the basis for a minimally invasive test of endometriosis. Hum Reprod 23: 1063-1068, 2008

15. Tamaresis JS, Irwin JC, Goldfien GA, Rabban JT, Burney RO, Nezhat C, DePaolo LV and Giudice LC: Molecular classification of endometriosis and disease stage using high-dimensional genomic data. Endocrinology 155: 4986-4999, 2014.

16. Muramatsu T and Miyauchi T: Basigin (CD147): A multifunctional transmembrane protein involved in reproduction, neural function, inflammation and tumor invasion. Histol Histopathol 18: 981-987, 2003.

17. Pitsos M and Kanakas N: The role of matrix metalloproteinases in the pathogenesis of endometriosis. Reprod Sci 16: 717-726, 2009.

18. Gilabert-Estellés J, Ramón LA, España F, Gilabert J, Vila V, Réganon E, Castelló R, Chirivella M and Estellés A: Expression of angiogenic factors in endometriosis: Relationship to fibrinolytic and metalloproteinase systems. Hum Reprod 22: 2120-2127, 2007

19. Ramón L, Gilabert-Estellés J, Castelló R, Gilabert J, España F, Romeu A, Chirivella M, Aznar J and Estellés A: mRNA analysis of several components of the plasminogen activator and matrix metalloproteinase systems in endometriosis using a real-time quantitative RT-PCR assay. Hum Reprod 20: 272-278, 2005.
20. Uzan C, Cortez A, Dufournet C, Fauvet R, Siffroi JP and Daraï E: Eutopic endometrium and peritoneal, ovarian and bowel endometriotic tissues express a different profile of matrix metalloproteinases-2, -3 and -11 , and of tissue inhibitor metalloproteinases-1 and -2. Virchows Arch 445: 603-609, 2004

21. Cominelli A, Gaide Chevronnay HP, Lemoine P, Courtoy PJ, Marbaix E and Henriet P: Matrix metalloproteinase-27 is expressed in CD163+/CD206+ M2 macrophages in the cycling human endometrium and in superficial endometriotic lesions. Mol Hum Reprod 20: 767-775, 2014

22. Zhao L, Gu C and Meng Y: Meta-analysis of the association between endometriosis and polymorphisms in ACE and PAI-1. Int J Clin Exp Med 9: 10602-10614, 2016.

23. Braza-Boïls A,Marí-Alexandre J,Gilabert J,Sánchez-Izquierdo D, España F, Estellés A and Gilabert-Estellés J: MicroRNA expression profile in endometriosis: Its relation to angiogenesis and fibrinolytic factors. Hum Reprod 29: 978-988, 2014.

24. Milde-Langosch K: The Fos family of transcription factors and their role in tumourigenesis. Eur J Cancer 41: 2449-2461, 2005.

25. Nemos C, Delage-Mourroux R, Jouvenot M and Adami P: Onset of direct 17-beta estradiol effects on proliferation and c-fos expression during oncogenesis of endometrial glandular epithelial cells. Exp Cell Res 296: 109-122, 2004.

26. Pan H, Sheng JZ, Tang L, Zhu R, Zhou TH and Huang HF: Increased expression of $\mathrm{c}$-fos protein associated with increased matrix metalloproteinase-9 protein expression in the endometrium of endometriotic patients. Fertil Steril 90: 1000-1007, 2008.

27. Morsch DM, Carneiro MM, Lecke SB, Araújo FC, Camargos AF, Reis FM and Spritzer PM: c-fos gene and protein expression in pelvic endometriosis: A local marker of estrogen action. J Mol Histol 40: 53-58, 2009.

28. O'Donovan KJ, Tourtellotte WG, Millbrandt J and Baraban JM: The EGR family of transcription-regulatory factors: Progress at the interface of molecular and systems neuroscience. Trends Neurosci 22: 167-173, 1999.

29. Birt JA, Nabli H, Stilley JA, Windham EA, Frazier SR and Sharpe-Timms KL: Elevated peritoneal fluid TNF- $\alpha$ incites ovarian early growth response factor 1 expression and downstream protease mediators: A correlation with ovulatory dysfunction in endometriosis. Reprod Sci 20: 514-523, 2013.

30. Djokovic D and Calhaz-Jorge C: Angiogenesis as a therapeutic target in endometriosis. Acta Med Port 27: 489-497, 2014.

31. Groothuis PG, Nap AW, Winterhager E and Grümmer R: Vascular development in endometriosis. Angiogenesis 8: 147-156, 2005.

32. Taylor RN, Yu J, Torres PB, Schickedanz AC, Park JK, Mueller MD and Sidell N: Mechanistic and therapeutic implications of angiogenesis in endometriosis. Reprod Sci 16: 140-146, 2009.

33. Bourlev V, Volkov N, Pavlovitch S, Lets N, Larsson A and Olovsson M: The relationship between microvessel density, proliferative activity and expression of vascular endothelial growth factor-A and its receptors in eutopic endometrium and endometriotic lesions. Reproduction 132: 501-509, 2006.

34. De Iaco P, Marabini A, Stefanetti M, Del Vecchio C and Bovicelli L: Acceptability and pain of outpatient hysteroscopy. J Am Assoc Gynecol Laparosc 7: 71-75, 2000.

35. Nisolle $M$, Paindaveine $B$, Bourdon $A$, Berlière $M$, Casanas-Roux F and Donnez J: Histologic study of peritoneal endometriosis in infertile women. Fertil Steril 53: 984-988, 1990. 